\title{
PROTEÍNAS RELACIONADAS CON LA DEFENSA EN LA INTERACCIÓN Sporisorium scitamineum - CAÑA DE AZÚCAR ${ }^{1}$
}

\author{
María La O-Hechavarría ${ }^{2}$,Ariel Arencibia-Rodríguez $z^{2}$, Rosemary López-Lee ${ }^{3}$, Ricardo Acevedo-Rojas ${ }^{2}$, Ondina \\ León-Díaz , Eida Rodríguez-Lema
}

\begin{abstract}
RESUMEN
Proteínas relacionadas con la defensa en la interacción Sporisorium scitamineum - caña de azúcar. El presente trabajo se realizó en el Centro Nacional de Sanidad Agropecuaria (CENSA), Habana, Cuba, durante el periodo 2001-2005, con el objetivo de determinar los patrones isoenzimáticos superóxido dismutasa y las secuencias de proteínas relacionadas con la defensa inducida en la interacción $S$. scitamineum - caña de azúcar, para lo cual fueron inoculadas con el patógeno las yemas de las variedades Ja60-5 (susceptible) y M31/45 (resistente) con una suspensión de esporidios del patógeno. La separación electroforética en condiciones nativas permitió la detección de dos isoformas ácidas de la enzima, una que se induce en mayor magnitud en la variedad resistente en comparación con la susceptible y otra que mantiene su nivel constitutivo para ambas variedades. El análisis ADNc-AFLP permitió la obtención de secuencias de PRs homólogas a glucanasas (PR2), quitinasas (PR3) y osmotina (PR5) inducidas diferencialmente en la variedad resistente. Los resultados obtenidos sugieren la participación de la enzima superóxido dismutasa en la señalización de la infección en esta interacción; así como la participación de PRs en la respuesta de resistencia inducida. Todo esto brinda la posibilidad de utilizar dichas proteínas como posibles marcadores bioquímicos y moleculares en Programas de Selección de Variedades Resistentes al Carbón de la Caña de Azúcar.
\end{abstract}

Palabras clave: Caña de azúcar, SOD, PRs, Sporisorium scitamineum, interacción.

\begin{abstract}
Defense related proteins in the interaction Sporisorium scitamineum - sugarcane. Laboratory experiments were conducted in the National Centre for Animal and Plant Health during 2001-2005. In this paper were determined superoxide dismutase iso-enzyme patterns and transcripts sequenced from PRs on the Sporisorium scitamineum - sugarcane interaction, for this reason were inoculated buds of both varieties Ja60-5 (susceptible) and M31/45 (resistant) with a suspension of pathogen teliospores. Native electrophoresis separation was detected two acidic isozymes, one was induced in high level in resistant varieties and the other at basal constitutive level in both varieties and differential displays sequence protein on resistant cultivar. The cADN-AFLP analysis permited to obtein sequences of PRs homologues to a glucanasas (PR2), quitinasas (PR3) and osmotina (PR5) diferentially induced on resistant variety. These results confirm that superoxido dismutase enzyme is involved in signal pathway on this interaction; and PRs induction on induced defence response and there are possible use as biochemical and molecular marker on the Sugarcane Breeding Program.
\end{abstract}

Key words: Sugar cane, SOD, PRs, Sporisorium scitamineum, interaction.

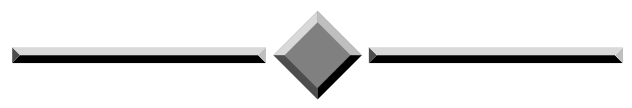

1 Recibido: 30 de noviembre, 2006. Aceptado: 29 de agosto, 2007. Estos resultados forman parte de la Tesis Doctoral "Contribución a la caracterización molecular de la interacción caña de azúcar-S. scitamineum".

2 Instituto Nacional de Investigaciones de la Caña de Azúcar (INICA), Carretera al Central Martínez Prieto Km 2 1/2. Boyeros, CP 19390. C. Habana, Cuba. Correo electrónico: lao@inica.minaz.cu, ariel.arencibia@inica.minaz.cu, acevedo@inica.minaz.cu y eida@inica.minaz.cu, respectivamente.

3 Centro Nacional de Sanidad Agropecuaria (CENSA), Apdo 10, San José de Las Lajas, La Habana, Cuba. Tel. 2624436. Correo electrónico: rlopez@censa.edu.cu y ondina@censa.edu.cu, respectivamente. 


\section{INTRODUCCIÓN}

La acumulación de aniones de oxígeno reactivos es fundamental durante la respuesta de las plantas a estrés biótico y abiótico. En ambas situaciones éstos pueden jugar un papel divergente: exacerbando los daños o activando múltiples respuestas defensivas, actuando como moléculas de señalización (Klarzynski et al. 2003; Compant et al. 2005; Ohno et al. 2006)). Por la citotoxicidad de el anión superóxido y del peróxido de hidrógeno se sugiere que ésta es la primera línea de defensa y en su metabolismo participa un grupo de enzimas, incluidas las superóxido dismutasas (SOD) (Pozos 1999; Foyer 2005); además de la posterior acumulación de proteínas relacionadas con la patogénesis (PR-1, PR-2, PR-3, PR-5, entre otras) como parte de la inducción de la resistencia sistémica adquirida (Roetschi et al. 2001; López 2002; Costales et al. 2006; Martínez et al. 2006).).

El agente causal del carbón (Sporisorium scitamineum) es una de las principales enfermedades que afecta al cultivo de la caña de azúcar, a nivel mundial. En el caso particular de la interacción de la caña de azúcar y Sporisorium scitamineum (Syd. M. Piepenbr., M. Stoll \& Oberw.), entre las investigaciones más recientes encaminadas a detectar los mecanismos que participan en la respuesta defensiva de la planta, se destacan los resultados de Heinze et al. (2001); Butterfield et al. (2004) y Borrás et al. (2005), quienes encontraron secuencias homólogas a genes que participan en el metabolismo de los fenilpropanoides y flavonoides, planteado por Lloyd y Naidoo (1983) sobre la participación de estos compuestos en la resistencia a $S$. scitamineum.

El objetivo de este trabajo fue la determinación de proteínas nativas SOD y secuencias de fragmentos derivados de transcriptos con homología a PRs presentes en extractos crudos de yemas de caña de azúcar de las variedades M31/45 (resistente) y Ja60-5 (susceptible) inoculadas con $S$. scitamineum.

\section{MATERIALES Y MÉTODOS}

Los experimentos de laboratorio se desarrollaron en las instalaciones del Centro Nacional de Sanidad
Agropecuaria (CENSA), Cuba, en el período del 20012005. Se utilizaron tercios medios de tallos de nueve meses de edad de las variedades Ja60-5 (susceptible) y M31/45 (resistente), los que se cortaron en porciones de yemas e inoculadas, 30 por cada variedad, levantando cuidadosamente la escama de la yema y depositando una gota de suspensión de esporidios de un aislamiento monospórico de $S$. scitamineum a una concentración de $1,5 \times 10^{7} \mathrm{ufc} / \mathrm{ml}$. Se dejó igual número de muestras como control sano. Las yemas se pusieron a germinar en incubadora a $30^{\circ} \mathrm{C}$ y $85 \%$ de HR en oscuridad.

Las muestras se tomaron a tiempo cero, 24 y 72 horas postinoculación, se procesaron tres réplicas en cada caso; a partir de $10 \mathrm{~g}$ de yemas germinadas homogeneizadas en nitrógeno líquido.

Para la extracción de proteínas totales se utilizó solución de acetato de sodio $0,1 \mathrm{M}$. La concentración de proteínas se determinó por el método de Bradford (1976), la lectura de la absorbancia a $595 \mathrm{~nm}$ se realizó en un espectrofotómetro (Ultrospec Plus Spectrophotometer, Pharmacia LKB) para lo cual se empleó una curva patrón de albúmina bovina (BSA) a partir de una solución madre de $1 \mathrm{mg} / \mathrm{ml}$.

La electroforesis en geles de poliacrilamida (PA), en condiciones no desnaturalizantes, se realizó en equipo de electroforesis vertical minigel (BIORAD), durante tres horas, con corriente constante de $30 \mathrm{~mA}$ a $25^{\circ} \mathrm{C}+/ 2{ }^{\circ} \mathrm{C}$; se utilizó solución amortiguadora de corrida: Tris-glicina $\mathrm{pH} 8,3$, empleando gel separador de PA $12 \%$ y $5 \%$ en el concentrador con separadores de $0,75 \mathrm{~mm}$, se aplicaron $30 \mu \mathrm{g}$ de proteínas totales de caña de azúcar. Para el revelado del zymograma SOD se utilizó como sustrato azul nitro-tetrazolio $2,45 \mathrm{mM}$ en solución fosfato de sodio $50 \mathrm{mM} \mathrm{pH} \mathrm{7,8} \mathrm{y} \mathrm{ribofla-}$ vina $28 \mathrm{mM}$, se observaron bandas blancas sobre fondo morado (Pozos 1999).

La purificación del RNA total se realizó por el sistema comerial PROMEGA. La síntesis del cDNA fue utilizando el sistema Universal RiboClone ${ }^{\circledR}$ (PROMEGA). Para el análisis cDNA-AFLP se siguió el procedimiento descrito por Bachem et al. (2000). El cDNA se digirió con las enzimas de restricción AseI y TaqI y para la reacción de PCR se utilizó la combinación de cebadores que se presenta en el Cuadro 1. A los productos de AFLP se les realizó electroforesis 
Cuadro 1. Cebadores utilizados en el análisis AFLP. Centro Nacional de Sanidad Agropecuaria (CENSA), Cuba, 2001-2005.

\begin{tabular}{llc}
\hline Cebadores & \multicolumn{1}{c}{ Secuencias } & NS* \\
\hline AseI-1 & 5'-GAC TGC GTA CCT AAT & at \\
AseI-2 & 5'-GAC TGC GTA CCT AAT & cg \\
AseI-3 & 5'-GAC TGC GTA CCT AAT & tg \\
TaqI-1 & 5'-GAT GAG TCC TGA CCG A & ta \\
TaqI-2 & 5'-GAT GAG TCC TGA CCG A & gt \\
TaqI-3 & 5'-GAT GAG TCC TGA CCG A & ca \\
\hline
\end{tabular}

* Nucleótidos selectivos.

en geles PA 9\% y se revelaron con tinción con plata (PROMEGA).

Las bandas polimórficas derivadas de transcriptos se cortaron e incubaron en solución amortiguadora TE (10 mM tris, pH 7,5 y $1 \mathrm{mM}$ EDTA, pH 8,0) $12 \mathrm{~h} \mathrm{a}$ $37^{\circ} \mathrm{C}$. Las bandas extraídas se utilizaron como molde para reamplificar por PCR utilizando los cebadores correspondientes para AFLP. Se les realizó secuenciación automática con el equipo y juegos de reactivos siguiente: Perkin-Elmer ABI PRISM Dye Terminator Cycle sequencing kit y ABI Model 377 DNA sequencer. Las secuencias se analizaron en el programa BLASTX (Altschul et al. 1997) y se compararon con las bases de datos GenBank, EMBL y DDBJ.

\section{RESULTADOS}

La separación electroforética en condiciones nativas permitió la detección de dos isoformas ácidas de superóxido dismutasa (Figura 1), una que se induce en mayor magnitud (SOD 1) en la variedad resistente en comparación con la susceptible y otra que mantiene su nivel constitutivo para ambas variedades (SOD 2).

En el Cuadro 2 se observan las secuencias de fragmentos derivados de transcriptos que se identificaron con homología a PR proteínas.

De las secuencias similares a PR proteínas, las PR-2 y PR-3 se inducen sólo en la variedad resistente a las 24 y/o 72 h, las identificadas como PR-5 se expresan, además, en la variedad susceptible a las 24 h (homóloga a Zea mays) y a las 72 h (homóloga a Saccharum sp.). La secuencia similar al gen poxN para peroxidasa es la única que se expresa a nivel constitutivo y se induce en mayor magnitud a las $24 \mathrm{~h}$ en la variedad susceptible y a las $72 \mathrm{~h}$ en la resistente.

\section{DISCUSIÓN}

En esta interacción los resultados concuerdan con una de las funciones de esta enzima en la planta, que debe de activarse o expresarse para regular el estrés oxidativo que conlleva cualquier ataque de patógenos (Roetschi et al. 2001; Ohno et al. 2006); pues el incremento se observó en las muestras de $24 \mathrm{~h}$ postinoculación con mayor intensidad; por lo que se pudiera decir que es una de las enzimas que participa en el evento de señalización de la respuesta defensiva, ya que coincide el marcado incremento de su actividad con el proceso de penetración del patógeno, el que se lleva a cabo de 6-36 h postinoculación (Alexander y Ramakisham 1980).

En otras interacciones (Pozos 1999; Solórzano 2002; Vandenabeele et al. 2003; Li et al. 2006) se destaca que las isoformas de SOD se pueden considerar un buen mecanismo de protección celular frente a la oxidación por los iones superóxido que pueden ser inducidos por diversas circunstancias como la senescencia, la acumulación de oxígeno, los procesos patogénicos y otras situaciones ambientales estresantes para la planta.

Los resultados aquí mostrados permiten establecer la posible relación del papel del gen poxN para peroxidasa y la explosión oxidativa en la respuesta de resistencia de la caña de azúcar contra el carbón. Este gen podría inducir otros transcriptos que se expresan diferencialmente en esta interacción, como las proteínas relacionadas con la defensa. Además, confirmaría los resultados de Damaj et al. (2005) sobre la alta coordinación entre las diferentes vías de señalización en caña de azúcar como respuesta a distintos tipos de estrés.

Las peroxidasas forman una compleja familia de proteínas que catalizan la reducción de varios sustratos que utilizan el $\mathrm{H}_{2} \mathrm{O}_{2}$. En particular, las dependientes del $\mathrm{pH}$ en la pared celular podrían ser la fuente de $\mathrm{H}_{2} \mathrm{O}_{2}$ en 

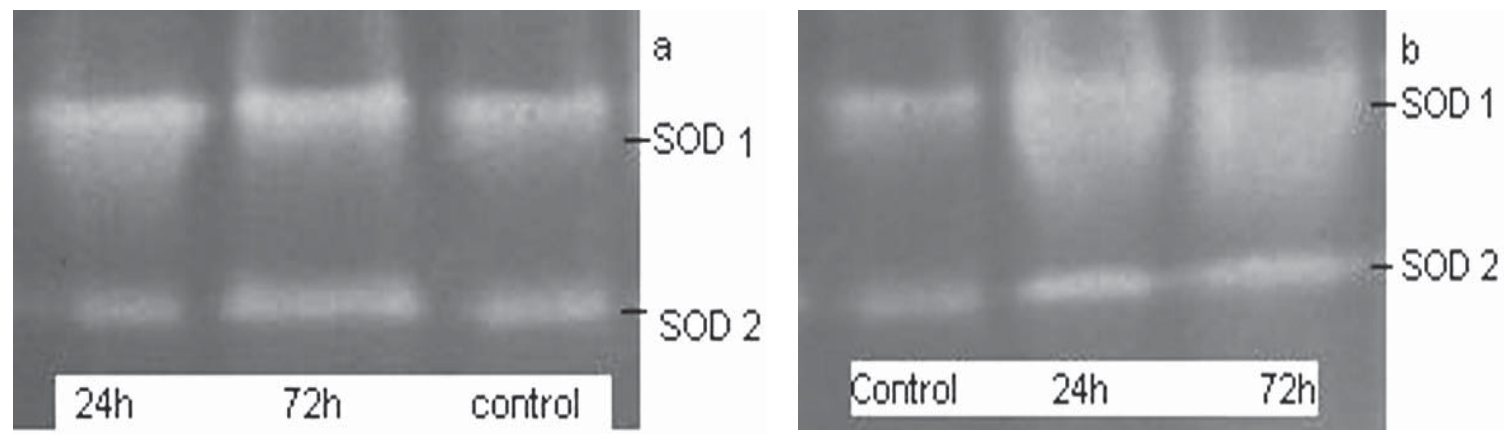

Figura 1. Patrón de acumulación temporal de isoenzimas de superóxido dismutasas (SOD) en la variedad susceptible Ja60-5 (a) y resistente M31/45 (b) posterior a la inoculación con S. scitamineum, a tiempo cero (C), 24 y $72 \mathrm{~h}$ postinoculación. Centro Nacional de Sanidad Agropecuaria (CENSA), Cuba. 2003.

Cuadro 2. Secuencias de fragmentos derivados de transcriptos expresados diferencialmente en la interacción S. scitamineum - caña azúcar. Cuba, 2001 - 2005.

\begin{tabular}{|c|c|c|c|c|c|c|c|c|c|}
\hline \multirow[t]{2}{*}{ (pb) } & \multirow{2}{*}{$\begin{array}{c}\text { Homología } \\
\text { (\# Acceso) }\end{array}$} & \multirow[t]{2}{*}{ Proteína } & \multirow[t]{2}{*}{ Valor $\mathbf{E}$} & \multicolumn{3}{|c|}{$\begin{array}{c}\text { Susceptible } \\
\text { Ja60-5 }\end{array}$} & \multicolumn{3}{|c|}{$\begin{array}{c}\text { Resistente } \\
\text { M31/45 }\end{array}$} \\
\hline & & & & $\mathbf{0}$ & 24 & 72 & $\mathbf{0}$ & 24 & 72 \\
\hline 392 & CF576358 & PR-2: Quitinasa (Saccharum sp.) & $1.8 \mathrm{e}-118$ & & & & & $\mathrm{x}$ & $\mathrm{x}$ \\
\hline 427 & Z99959 & PR-2: Quitinasa (Musa acuminata) & $4.3 e-32$ & & & & & & $\mathrm{x}$ \\
\hline 380 & BU925830 & Similar a PR-3: ß-glucanasa (Saccharum sp.) & $3.6 \mathrm{e}-120$ & & & & & $\mathrm{x}$ & $\mathrm{x}$ \\
\hline 377 & U82201 & PR-5 proteína (Zea mays) & $3.6 e-29$ & & $\mathrm{x}$ & & & $\mathrm{x}$ & $\mathrm{x}$ \\
\hline 580 & D49551 & $\begin{array}{l}\text { gen poxN para peroxidasa, exon1-4 (Oryza } \\
\text { sativa- cultivar japonica) }\end{array}$ & $9.5 \mathrm{e}-30$ & $\mathrm{x}$ & $\mathrm{X}$ & $\mathrm{x}$ & $\mathrm{x}$ & $\mathrm{x}$ & $\mathrm{X}$ \\
\hline 246 & D25236 & PR-3: ß- 1,3-1,4 Glucanasa (Oryza sativa) & $7.2 \mathrm{e}-61$ & & & & & & $\mathrm{x}$ \\
\hline 322 & CF570197 & Similar a PR-5: osmotina (Saccharum sp.) & $1.3 \mathrm{e}-82$ & & & $\mathrm{x}$ & & $\mathrm{x}$ & $\mathrm{x}$ \\
\hline 258 & CD347683 & $\begin{array}{l}\text { Similar al precursor Endo-1,3;1,4- } 3 \text {-D-gluca- } \\
\text { nasa (Oryza minuta) }\end{array}$ & $6.1 \mathrm{e}-56$ & & & & & & $\mathrm{x}$ \\
\hline
\end{tabular}

el apoplasto, en presencia de sustancias reducidas liberadas por las células dañadas (Torres et al. 2006).

Los aniones reactivos de oxígeno son moléculas muy versátiles que participan en diversas respuestas celulares, incluida la muerte celular programada, el desarrollo, el gravitropismo y las señales hormonales (Kwak et al. 2006). Además, son importantes señales de activación de genes de defensa y poseen funciones reguladoras de la misma en conjunto con otras moléculas señalizadoras, particularmente con el ácido salicílico y el oxido nítrico (Del Río et al. 2006; Wang y Higgins 2006).
Aunque la explosión oxidativa primaria ocurre en el apoplasto posterior al reconocimiento del patógeno, los aniones reactivos de oxígeno producidos en otros compartimentos celulares, también podrían tener funciones en la defensa. Varios sistemas de eliminación de aniones reactivos de oxígeno, incluidas las ascorbato peroxidasas, superóxido dismutasas y catalasas mantienen la homeostasis en los diferentes compartimentos celulares en las plantas (Reumann et al. 2004; Küpper et al. 2006; Del Río et al. 2006).

La inducción muy temprana de PRs puede jugar un papel importante en las reacciones de defensa, lo cual 
se refiere en muchos patosistemas: Alternaria solanitomate (Solórzano 2002), Puccinia melanocephalacaña de azúcar (López 2002), Phytophthora megasperma- tabaco (Cordelier et al. 2003), entre otros.

Las PRs 2 y 3 que poseen funciones hidrolíticas pueden desempeñar funciones protectoras directas, al hidrolizar la pared celular de los patógenos, o indirecta, en los mecanismos de defensa de las plantas, al liberar elicitores que estimulan la acumulación de fitoalexinas, deposición de lignina, etc. (Faure 2002; Van Loon et al. 2006).

En el caso particular de S. scitamineum por la constitución química de su pared celular, rica en glucanos y quitina (Banks et al. 2005), las glucanasas y quitinasas pueden degradar directamente dicha estructura y por tanto limitar su crecimiento.

De forma general, a partir de los resultados obtenidos, se ratifica que la acumulación de las PR proteínas no está restringida únicamente a las plantas resistentes, sino que también se pueden detectar en interacciones compatibles. Sin embargo, el tiempo de aparición y la magnitud de la inducción puede diferir, lo cual varios autores señalan que influye en la reacción de defensa (Bulow et al. 2004; Compant et al. 2005).

\section{CONCLUSIONES}

Los resultados obtenidos confirman la activación de las enzimas superóxido dismutasas y proteínas relacionadas con la patogénesis (PRs) en esta interacción, todo lo cual es la primera vez que se estudia en esta enfermedad. La marcada diferencia de expresión entre la variedad susceptible y resistente, indica que estas enzimas puedan ser utilizadas como posibles marcadores bioquímicos y moleculares en Programas de Selección de Variedades Resistentes al Carbón de la Caña de Azúcar.

\section{LITERATURA CITADA}

ALEXANDER, K.C.; RAMAKISHAM, K. 1980. Infection of the bud, establishment in the host and production of whips in sugarcane (Ustilago scitaminea). Proc. Int. Soc. Sug. Technol. 17: 1453-1455.
ALTSCHUL, S.F.; MADDEN, T.L.; SCHAFFER, A.A.; ZHANG, J.H.; ZHANG, Z.; MILLER, W.; LIPMAN, D.J. 1997. Gapped BLAST and PSIBLAST: A new generation of protein database search programs, Nucleic Acids Res 25: 3389-3402.

BACHEM, C.W.B.; VAN DER HOEVEN, R.S.; LUCKER, J.; OOMEN, R.J.F.; CASARINI, E.; JACOBSEN, E.; VISSER R.G.F. 2000. Functional genomic analysis of potato tuber life-cycle. Potato Res. 43:297-312.

BANKS, I.R.; SPECHT, C.C.; DONLIN, M.J.; GERIK, K.J.; LEVITZ, S.M; LODGE, J.K. 2005. A chitin synthase and its regulators protein are critical for chitosan production and growth of the fungal pathogen Cryptococcus neoformans. Eucaryotic Cells: 4 (11): 1902-1912.

BORRÁS, O.; THOMMA, B.P.; CARMONA, E.; BORROTO, C.J.; PUJOL, M.; ARENCIBIA, A.; LÓPEZ, J. 2005. Identification of sugarcane genes induced in disease-resistant somaclones upon inoculation with Ustilago scitaminea or Bipolaris sacchari. Plant Physiol. and Biochem. 43:1115-1121.

BRADFORD, M.M. 1976. A rapid and sensitive method for the quantification of microgram quantities of protein utilizing the principle of protein dye binding. Anal. Biochem. 72: 248-254.

BULOW, L.; SCHINDLER. M.; CHOI, C.; HEHL, R. 2004. PathoPlant ${ }^{\circledR}$ : A database on plant-pathogen interactions in silico biology bioinformation systems e.V 4: 00-44.

BUTTERFIELD, M.K.; RUTHERFORD, R.S.; CARSON, D.L.; HUCKETT, B.I. 2004. Application of gene discovery to varietal improvement in sugarcane, South Afri. J. Bot. 70: 167-172.

COMPANT, S.; DUFFY, B.; NOWAK, J.; CLÉMENT, C.; BARKA, E. 2005. Use of plant growth-promoting bacteria for biocontrol of plant diseases: principles, mechanisms of action, and future prospects. Minireview Applied and Environmental Microbiology 71 (9): 4951-4959.

CORDELIER, S.; DE RUFFRAY, P.; FRITIG, B.; KAUFFMANN, S. 2003. Biological and molecular comparison between localized and systemic acquired resistance induced in tobacco by a Phytophthora

AGRONOMÍA MESOAMERICANA 18(2): 197-203. 2007 
megasperma glycoprotein elicitin. Plant Mol. Biol. 51 (1): 109-118.

COSTALES, D.; CABRERA J.C.; FALCÓN, A. 2006. Efecto de una mezcla de oligosacáridos pécticos en la inducción de resistencia en plántulas de tabaco contra Phythophthora nicotianae. XV Congreso Científico INCA. ISBN 959-7023-36-9.157p.

DAMAJ, M.; BEREMAND, P.P.; AL-DOURI, A.F.; SILVA, M.A.; JIFON, J.L.; KUMPATLA, S.P.; REDDY, A.S.; THOMAS, T.L.; MIRKOV, T.E. 2005. Coordinated defense responses in sugarcane revealed by transcriptional profiling. Abstracts of the PlantPathogen Interaction Workshop. Plant \& Animal Genomes XIII Conference. W158: Intl. Consortium for Sugarcane Biotech. (ICSB). San Diego.

DELRÍO, L.A.; SANDALIO, L.M.; CORPAS, F.J.; PALMA, J.M.; BARROSO, J.B. 2006. Reactive oxygen species and reactive nitrogen species in peroxisomes. Production, scavenging, and role in cell signalling. Plant Physiol. 141:330-335.

FAURE, D. 2002. The family 3-glycoside hydrolases: from housekeeping functions to host-microbe interactions. Applied and Environmental Microbiology 68(4):14851490 .

FOYER, C.H. 2005. Redox homeostasis and antioxidant signalling: a metabolic interface between stress perception and physiological responses. The Plant Cell 17:1866-1875.

HEINZE, B.S.; THOKOANE, L.N.; WILLIAMS, N.J.; BARNES, J.M.; RUTHERFORD, R.S. 2001. The smut- sugarcane interaction as a model system for the integration of marker discovery and gene isolation. Proc. S. Afr. Sug. Technol. Ass. 75: 88-93.

KLARZYNSKI, O.; DESCAMPS, V.; PLESSE, B.; YVIN, J.C.; KLOAREG, B.; FRITIG, B. 2003. Sulfated fucan oligosaccharides elicit defense responses in tobacco and local and systemic resistance against tobacco mosaic virus. Mol. Plant Microbe Interact. 16 (2): 115-112.

KÜPPER, F.; EMMANUEL, G.; BONEBERG, E.; MORATH, S.; SALAÜN, J.; POTIN, P. 2006. Early events in the perception of lipopolysaccharides in the brown alga Laminaria digitata include an oxidative burst and activation of fatty acid oxidation cascades. J. Exp. Bot. 57(9):1991-1999.

KWAK, J.M.; NGUYEN, V.; SCHROEDER, J. 2006. The role of reactive oxygen species in hormonal responses. Plant Physiol. 141:323-329.

LI, J.; ZHANG Z-G, JI. R.; WANG, Y-C.; ZHENG X-B. 2006. Hydrogen peroxide regulates elicitor PB90induced cell death and defense in non-heading chinese cabbage. Physiological and molecular plant pathology 67: 220-230.

LLOYD, H.L.; NAIDOO, M. 1983. Chemical assay potentially suitable for determination of smut resistance of sugarcane cultivars. Plant Disease 67: 1103-1105.

LÓPEZ, R. 2002. Bases bioquímicas moleculares de la respuesta de defensa de la caña de azúcar (Saccharum spp.) a Puccinia melanocephala H \& P. Sydow. Tesis en opción al grado de Doctor en Ciencias Agrícolas. CENSA. La Habana. 118 p.

MARTÍNEZ,M.A;TORRES, G.;CABRERA,J.C.;VARGAS, I. 2006. Inducción de proteínas relacionadas con la patogénesis por la aplicación de oligogalacturónidos bioactivos en plantas de vid (Vitis vinifera $\mathrm{L}$.) y tabaco (Nicotiana tabacum L.). XV Congreso Científico INCA. ISBN 959-7023-36-9.155 p.

OHNO, S.; IM, H.; KNUDSON, C.; KNUDSON, J. 2006. Hyaluronan Oligosaccharides Induce Matrix Metalloproteinase 13 via Transcriptional Activation of NFB and p38 MAP Kinase in Articular Chondrocytes J. Biol. Chem. 281 (26): 17952-17960.

POZOS, J.M. 1999. Inducción de enzimas hidrolíticas en raíces de tomate (Lycopersicum sculentum) como respuesta a la formación de MA y su implicación en el control biológico de $O$. parasitica. Tesis doctoral. Univ. Granada. Fac. Ciencias. 100 p.

REUMANN, S.; LEMKE, M.; BABUJEE, S. 2004. AraPerox: a database of putative Arabidopsis proteins from plant peroxisomes. Plant Physiol. 136: 2587-2608.

ROETSCHI, A.; SI-AMMOUR, A.; BELBAHRI, L.; MAUCH, F.; MAUCH-MANI, B. 2001. Characterization of an Arabidopsis- Phytophthora pathosystem: resistance requires a functional PAD2

AGRONOMÍA MESOAMERICANA 18(2): 197-203. 2007 
gene and is independent of salicylic acid, ethylene and jasmonic acid signalling. Plant J. 28:293-305.

SOLÓRZANO, E. 2002. Estudio de las enzimas y proteínas de defensa en la interacción tomate (Lycopersicon esculentum, Mill) vs tizón temprano (Alternaria solani Ellis y Martín (Jones y Grout)). Tesis para optar por el grado de Doctor en Ciencias Agrícolas. CENSA. Habana. 124 p.

TORRES, M.; JONES, J.D.G.; DANGL, J.L. 2006. Reactive oxygen species signalling in response to pathogens. Plant Physiol. 141: 373-378.

VAN LOON, L.C.; REP, M.; PIETERSE, C.M.J. 2006. Significance of inducible defence-related proteins in infected plants. Ann Rev Phytopathol., 44: 7.1-7.28.
VANDENABEELE, S.; VAN DERKELEN, K.; DAT, J.; GADJEV, J.; BOONEFAES, T.; MORSA, S.; ROTTIERS, P.; SLOOTEN, L.; VAN MONTAGU, M.; ZABEAU, M.; INZE, D.; VAN BREUSEGEM, F. 2003. A comprehensive analysis of hydrogen peroxide induced gene expression in tobacco. Proc. Natl. Acad. Sci. USA. 100 (26): 16113-18118.

WANG, J.; HIGGINS, V.J. 2006. Nitric oxide modulates $\mathrm{H}_{2} \mathrm{O}_{2}$-mediated defences in the Colletotrichum coccodes-tomato interaction. Physiol. and Mol. Plant Pathol. 67: 131- 137. 